\title{
Amniotic lamellar body count and congenital diaphragmatic hernia in humans and in a rat model
}

\author{
Yuriko Watanabe', Hiroyuki Tsuda', Tomomi Kotani', Seiji Sumigama', Yukio Mano', Masahiro Hayakawa², Yoshiaki Sato² \\ and Fumitaka Kikkawa'
}

BACKGROUND: We examined the extent of fetal lung hypoplasia and lung maturation using the amniotic lamellar body count (LBC) in congenital diaphragmatic hernia (CDH).

METHODS: We obtained 30 amniotic fluid samples from human $\mathrm{CDH}$ cases during cesarean section. We assessed LBC, magnetic resonance imaging $(\mathrm{MRI})$, and ultrasound findings for predicting the prognosis of $\mathrm{CDH}$. We collected newborn amniotic fluid and lung tissue at embryonic day (E)21 from normal and nitrofeninduced $\mathrm{CDH}$ rats (administered $100 \mathrm{mg}$ orally at E9). Amniotic LBCs in rats were measured using light microscopy.

RESULTS: In human CDH, LBC was significantly higher in the surviving than in the deceased group $(P<0.01)$. A significant positive correlation was observed between LBC and percentage of fetal lung volume on MRI $(P<0.001 ; r=0.716)$. In rats, LBC was significantly higher in controls than in $\mathrm{CDH}$ rats $(P<$ 0.01) and correlated with fetal lung weight.

CONCLUSION: We conclude that $L B C$ is useful for predicting lung hypoplasia in human $\mathrm{CDH}$ after 35 gestational weeks and in a rat model of nitrofen-induced $\mathrm{CDH}$.

C ongenital diaphragmatic hernia $(\mathrm{CDH})$ is a developmental discontinuity of the diaphragm that affects one in 2,5005,000 live births (1), resulting in pulmonary hypoplasia and pulmonary hypertension, which are often fatal. To improve clinical care and counseling, reliable prenatal parameters predicting fetal outcome in $\mathrm{CDH}$ are urgently needed. Prenatal predictors of fetal outcome in $\mathrm{CDH}$ have been reported to be focused mainly on the assessment of fetal lung volume (FLV). Ultrasound and magnetic resonance imaging (MRI) have been used to measure antenatal FLV and to qualify fetal pulmonary hypoplasia, but a gold-standard parameter has not yet been established.

The lamellar body count (LBC) was first described in 1989 (2). Lamellar bodies are made up of a surfactant-containing lamellated structure and are secreted by type II pneumocytes (3). The similarity of lamellar body size to platelet size permits the use of a standard automated hematologic cell counter to quantify the number of lamellar bodies in amniotic fluid (4). Thus, the LBC is used to predict the degree of fetal lung maturity (FLM). Several studies have shown the LBC to accurately predict FLM (5-7). The LBC can be measured quickly and inexpensively, such that it is equal to or even better than the lecithin-to-sphingomyelin ratio for predicting the occurrence of respiratory distress syndrome (7).

In the current study, we hypothesized that if fetal pulmonary hypoplasia occurs because of $\mathrm{CDH}$, the number of amniotic lamellar bodies secreted from the fetal lung into the amniotic cavity would be small. Therefore, we examined the LBC value in $\mathrm{CDH}$ neonates and confirmed it to be a useful predictor of $\mathrm{CDH}$ severity. Furthermore, given that LBC was originally a predictor of FLM, in the current study we also examined whether fetal lung maturation in $\mathrm{CDH}$ neonates is equivalent to that in normal neonates, using a rat $\mathrm{CDH}$ model.

\section{RESULTS}

Registration of Clinical CDH Cases

We initially enrolled 30 cases diagnosed with $\mathrm{CDH}$ prenatally (Figure 1). We excluded 11 cases (delivery before 35 weeks: $n=$ 5; amniotic sampling failure: $n=3$; trisomy $18: n=2$; neonatal death due to severe congenital heart disease: $n=1$ ). Therefore, 19 cases were ultimately analyzed. We divided the newborns into two groups: infants who survived $(n=16)$ and those who $\operatorname{died}(n=3)$.

\section{Prognostic Findings for $\mathrm{CDH}$ in Humans}

Background and prenatal findings of our $\mathrm{CDH}$ cases are shown in Table 1. LBC was significantly higher in the surviving group than in the deceased group $\left(1.6 \pm 0.7 \times 10^{4} / \mu \mathrm{l}\right.$ vs. $0.5 \pm 0.3 \times 10^{4} /$ $\mu \mathrm{l} ; P<0.01)$. The percentage of FLV on MRI was also significantly higher in the surviving than in the deceased group $(36.6 \pm 27.2$ $\times 10^{4} / \mu \mathrm{l}$ vs. $\left.11.3 \pm 6.14 \times 10^{4} / \mu \mathrm{l} ; P<0.05\right)$. We assessed $\mathrm{LBC}$ and percentage of FLV as potentially useful parameters for predicting neonatal nitric oxide (NO) $(n=12)$ and extracorporeal membrane oxygen $(\mathrm{ECMO})(n=4)$ requirements in the surviving group $(n=16)$. LBC was significantly lower in infants needing $\mathrm{NO}$ and ECMO therapies than in those not needing such therapies $\left(1.5 \pm 0.6 \times 10^{4} / \mu \mathrm{l}\right.$ vs. $2.4 \pm 0.4 \times 10^{4} / \mu \mathrm{l}$ in NO; $P=0.010$, and $1.2 \pm 0.4 \times 10^{4} / \mu \mathrm{l}$ vs. $1.9 \pm 0.6 \times 10^{4} / \mu \mathrm{l}$ in ECMO; $P=0.049$, respectively). The percentage of FLV on MRI was significantly lower in infants needing $\mathrm{NO}$ and ECMO therapies than in those not needing such therapies $(24.4 \pm 11.7$ vs. $73.2 \pm 28.3$ for NO; 


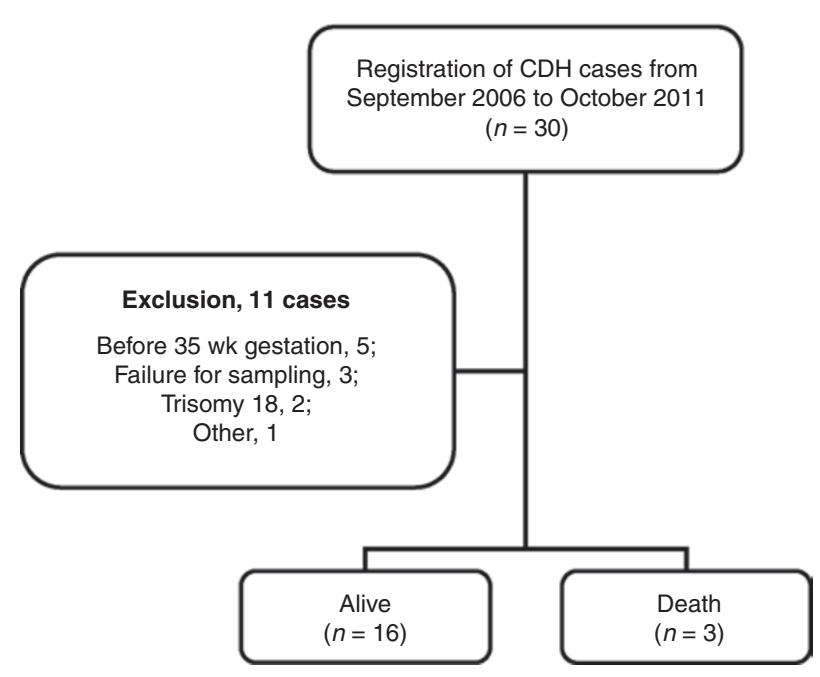

Figure 1. Algorithm for inclusion and exclusion of cases in this study. "Other" refers to a case of neonatal death due to severe congenital heart disease. $\mathrm{CDH}$, congenital diaphragmatic hernia.

Table 1. Background and prenatal findings of congenital diaphragmatic hernia in humans

\begin{tabular}{|c|c|c|c|}
\hline & $\begin{array}{c}\text { Patients who } \\
\text { survived }(n=16)\end{array}$ & $\begin{array}{l}\text { Patients who died } \\
\qquad(n=3)\end{array}$ & $P$ value \\
\hline Maternal age $(y)^{a}$ & $29.0 \pm 4.7$ & $31.7 \pm 2.1$ & 0.357 \\
\hline GA at delivery (weeks) & $37.44 \pm 0.13$ & $36.33 \pm 0.67$ & 0.054 \\
\hline Birth weight $<2,500 \mathrm{~g}^{\mathrm{b}}$ & $2 / 16(12.5 \%)$ & $0 / 3(0 \%)$ & 0.517 \\
\hline Male $^{\mathrm{b}}$ & $11 / 16(69 \%)$ & $2 / 3(67 \%)$ & 0.943 \\
\hline $\mathrm{DM}^{\mathrm{b}}$ & $0 / 16(0 \%)$ & $0 / 3(0 \%)$ & NA \\
\hline $\mathrm{PIH}^{\mathrm{b}}$ & $0 / 16(0 \%)$ & $0 / 3(0 \%)$ & NA \\
\hline FGR ${ }^{b}$ & $1 / 16(6 \%)$ & $0 / 3(0 \%)$ & 0.517 \\
\hline GA at diagnosis (wks) ${ }^{\mathrm{a}}$ & $28.6 \pm 3.3$ & $25 \pm 1.0$ & 0.086 \\
\hline $\operatorname{LBC}\left(10^{4} / \mu \mathrm{l}\right)^{\mathrm{a}}$ & $1.6 \pm 0.7$ & $0.5 \pm 0.3$ & 0.009 \\
\hline LHR $^{a}$ & $1.39 \pm 0.59$ & $1.20 \pm 0.40$ & 0.233 \\
\hline FLV on MRI $\left(\mathrm{cm}^{3}\right)^{\mathrm{a}}$ & $15.4 \pm 11.2$ & $7.28 \pm 2.42$ & 0.240 \\
\hline $\begin{array}{l}\text { Percentage of FLV on } \\
\mathrm{MRI}^{\mathrm{c}}\end{array}$ & $36.6 \pm 27.2$ & $11.3 \pm 6.14$ & 0.034 \\
\hline Liver herniation ${ }^{\mathrm{b}}$ & $7 / 16(44 \%)$ & $3 / 3(100 \%)$ & 0.466 \\
\hline Positive $\mathrm{CPB}^{\mathrm{b}}$ & $14 / 16(88 \%)$ & $0 / 3(0 \%)$ & 0.084 \\
\hline Polyhydramnios ${ }^{\mathrm{b}}$ & $3 / 16(19 \%)$ & $3 / 3(100 \%)$ & 0.222 \\
\hline
\end{tabular}

$\mathrm{CPB}$, contralateral pulmonary baseline on MRI; DM, diabetes mellitus; FGR, fetal growth restriction; FLV, fetal lung volume; GA, gestational age; LBC, lamellar body count; LHR, lung-to-head ratio; MRI, magnetic resonance imaging; NA, not available; $\mathrm{PIH}$, pregnancy-induced hypertension.

aStudent's $t$-test was used. ${ }^{\text {}} \chi^{2}$ test was used. cMann-Whitney $U$ test was used.

$P=0.002$, and $13.9 \pm 7.5$ vs. $44.2 \pm 27.2$ for ECMO; $P=0.008$, respectively). Other prenatal predictors including gestational age at diagnosis, lung to head ratio (LHR), absolute FLV, liver herniation, and polyhydramnios did not significantly impact the outcomes of CDH infants. The rates of diabetes mellitus, preeclampsia, and fetal growth restriction did not differ significantly between the two groups. Furthermore, we analyzed the relationship between LBC and percentage of FLV (Figure 2). A significant positive correlation was observed $(P<0.001 ; r=0.716)$.

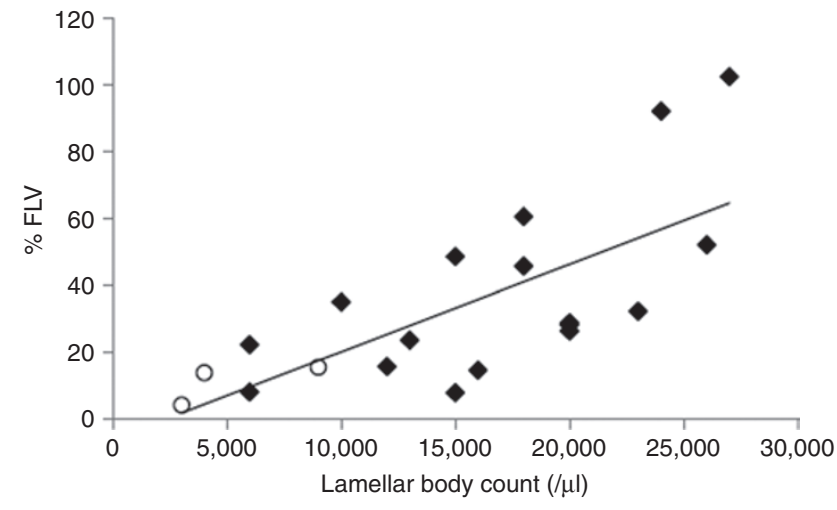

Figure 2. Scattergram showing the relationship between percentage of FLV and lamellar body count in human CDH. A significant positive correlation was observed $(P<0.001 ; r=0.716)$. Filled diamonds indicate patients who survived, open circles indicate patients with $\mathrm{CDH}$ who died. $\mathrm{CDH}$, congenital diaphragmatic hernia; FLV, fetal lung volume.
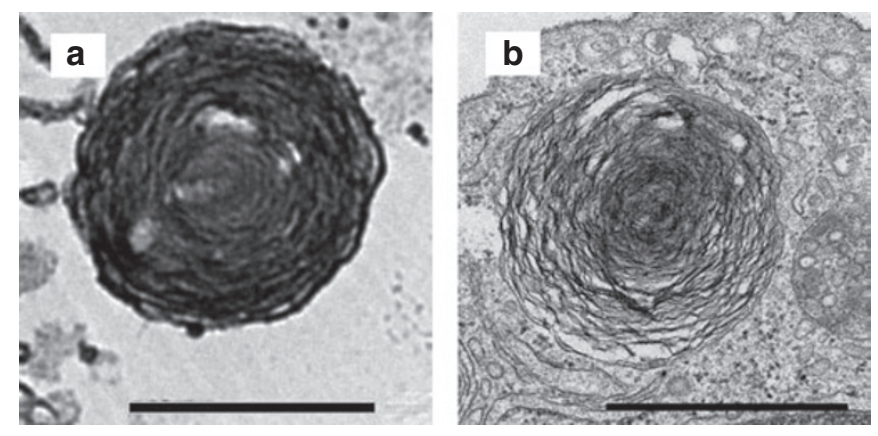

Figure 3. Electron microscopy of a lamellar body. Lamellar bodies are detected in (a) amniotic fluid and (b) type II alveolar cells. The lamellar body sizes were similar, at $\sim 1 \mu \mathrm{m}$, in the two sites. Bars $=1.0 \mu \mathrm{m}$.

\section{LBC and Lung Weight in a Rat CDH Model}

In the rat experiment, we collected amniotic fluid and lung tissue from newborns at embryonic day (E)21 and confirmed the presence of lamellar bodies in both the amniotic fluid (Figure 3a) and type II alveolar cells of the lung (Figure 3b) using electron microscopy. We also confirmed a similar lamellar body size in amniotic fluid and type II alveolar cells, of approximately $1-2 \mu \mathrm{m}$, comparable to the lamellar body findings in human amniotic fluid $(2,8)$. Furthermore, after exposure of pregnant rats to a 100-mg dose of nitrofen, $48.8 \%$ (61 of 125) of newborns had diaphragmatic hernias. LBC was significantly higher in control rats than in $\mathrm{CDH}$ rats $(3,527 \pm 211 / \mu \mathrm{l}$ vs. $1,564 \pm 358 / \mu \mathrm{l} ; P<0.01$ ) (Figure $4 \mathrm{a}$ ). Furthermore, lung weight was also significantly higher in controls than in $\mathrm{CDH}$ newborn rats $(0.143 \pm 0.024 \mathrm{~g}$ vs. $0.071 \pm 0.022 \mathrm{~g} ; P<0.01)$ (Figure $4 b$ ). In addition, the LBC per unit lung weight in controls was similar to that in $\mathrm{CDH}$ rats $\left(25.3 \pm 1.59 \times 10^{3} / \mu \mathrm{l}\right.$ vs. $\left.34.6 \pm 15.2 \times 10^{3} / \mu \mathrm{l} ; P=0.544\right)$.

\section{Western Blotting for ABCA3 Expression in Fetal Rat Lung}

We examined the expression of $\mathrm{ABCA} 3$ in lung tissues from newborn rats, delivered at E21, by western blotting (Figure 5a). ABCA3 expression did not differ significantly between controls and $\mathrm{CDH}$ rats $(P=0.551)$ (Figure $5 \mathrm{~b})$. 
a

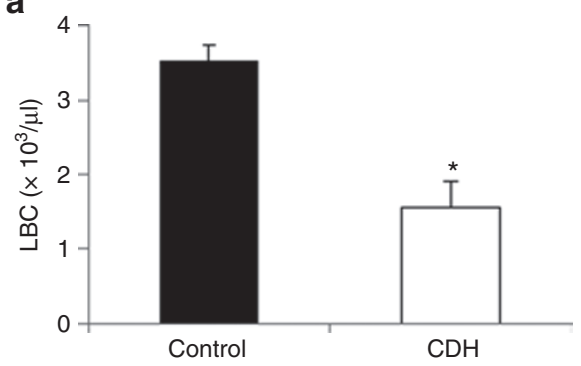

b

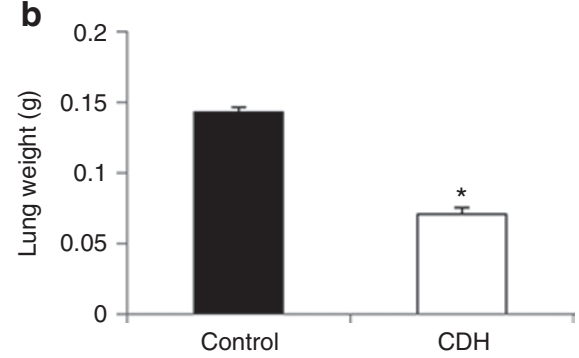

Figure 4. Amniotic fluid $L B C$ and lung weights in newborn rats. (a) $L B C$ was significantly higher in controls than in $\mathrm{CDH}$ rats $(3,527 \pm 211 / \mu \mathrm{l}$ vs. $1,564 \pm 358 / \mu \mathrm{l} ; P<0.01$ ). (b) Furthermore, newborn lung weights were significantly higher in controls than in CDH rats $(0.143 \pm 0.024 \mathrm{~g}$ vs. $0.071 \pm$ $0.022 \mathrm{~g} ; P<0.01)$. ${ }^{*} P<0.01$. Bars represent $S E M$. Controls are normal pups. $\mathrm{CDH}$, congenital diaphragmatic hernia; LBC, lamellar body count.

\section{a}

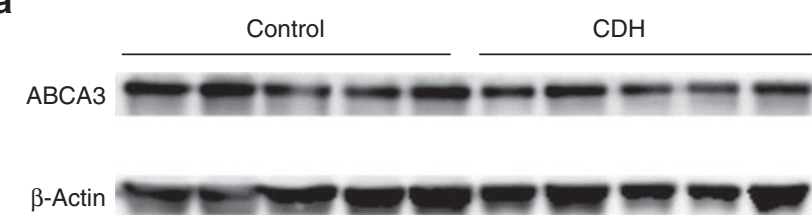

b

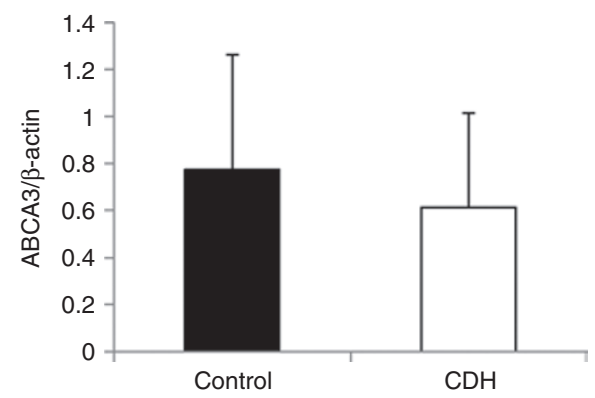

Figure 5. $A B C A 3$ and $\beta$-actin expressions in rat lung tissue. (a) Western blotting and (b) the ratio of $A B C A 3$ expression to that of $\beta$-actin revealed that $A B C A 3$ expression did not differ significantly between controls and $C D H$ rats $(P=0.551)$. Bars represent SEM. Controls are normal pups. $A B C A 3, A T P$-binding cassette transporter $A 3 ; C D H$, congenital diaphragmatic hernia.

\section{DISCUSSION}

Pulmonary hypoplasia accounts for significant mortality and morbidity in neonates with $\mathrm{CDH}$. Therefore, a precise predictor of FLV is required. In the current study, we found that LBC and percentage of FLV on MRI differed significantly between surviving and deceased CDH cases. Conversely, LHR did not differ significantly. LHR is reportedly useful for predicting subsequent survival (9), but some recent studies could not confirm the prognostic value of LHR for survival or the necessity of ECMO therapy $(10,11)$. In general, ultrasonography has certain disadvantages. Image resolution is sometimes limited by fetal position, maternal obesity, and oligohydramnios, which result in data variability among examiners. This can make it difficult to distinguish between fetal lung and liver on ultrasound. The FLV prediction using MRI has been reported to be accurate $(12,13)$, but absolute FLV alone is not predictive of outcome because increasing lung volume with increasing gestational age could be a confounding factor (14). Therefore, percentage of FLV on MRI is considered to be a more useful predictor in $\mathrm{CDH}$ (15). In the current study, percentage of FLV on MRI, not absolute FLV, differed significantly in terms of the prediction of mortality in $\mathrm{CDH}$. We examined factors such as liver herniation, complete contralateral pulmonary baseline on MRI, and polyhydramnios but found no significant differences, although positive findings for these factors correlated with a poor outcome (death in all cases).

Amniotic LBC is reportedly an accurate predictor of FLM and has the advantage of being able to be determined as an absolute value on the basis of objective application of a cell counter. In the current study, we found that LBC correlated significantly with neonatal mortality in $\mathrm{CDH}$ after 35 gestational weeks. Moreover, LBC correlated closely with percentage of FLV on MRI. From this viewpoint, we hypothesize that LBC might be useful not only as a predictor of mortality but also as a predictor of hypoplastic lung in $\mathrm{CDH}$ after 35 gestational weeks. The lamellar body is a surfactant-containing lamellated structure that is secreted by type II pneumocytes (3). Therefore, LBC is used to estimate surfactant production in utero and, if lung maturation has been achieved in the fetus, to predict the extent of FLV.

Several studies have shown that diaphragmatic defects could be induced by administering a single 100-mg dose of nitrofen to pregnant rats between E8 and E11, and most significantly, that the defects produced were remarkably similar to those documented in human Bochdalek $\mathrm{CDH}$ with respect to their size, location, and accompanying intrusion of the abdominal viscera into the thoracic cavity (16-18). We administered a single 100-mg dose of nitrofen at E9 and confirmed the diaphragm defects in $48 \%$ of the rat pups. This rate is acceptable as compared with those in other reports $(19,20)$.

In a rat model, we confirmed the presence of lamellar bodies in amniotic fluid as well as in alveolar type II cells using electron microscopy; we also confirmed that the size of these lamellar bodies was similar to that in humans. In human samples, we were able to examine amniotic LBCs using a hematologic cell counter in our hospital, but we could not apply this device to examine the lamellar bodies in the rat model. Hence, we counted rat lamellar bodies by light microscopy. LBCs determined by light microscopy reportedly correlate well with results obtained using standard hematologic cell counter techniques in human samples (21). This is the first report demonstrating amniotic LBCs in a rat model.

In our $\mathrm{CDH}$ rat model, fetal lung weight was significantly decreased, and amniotic LBC was also significantly decreased 
as compared with controls. The LBC per unit lung weight in $\mathrm{CDH}$ was similar to that in controls. Therefore, we can conclude that amniotic LBCs show a good correlation with fetal lung weight in $\mathrm{CDH}$ rat models, similar to the human $\mathrm{CDH}$ results. Furthermore, we examined $\mathrm{ABCA} 3$ expression by western blotting to assess fetal lung maturation in the rat. ABCA3 protein is expressed predominantly at the limiting membranes of the lamellar bodies in alveolar type II cells (22) and plays an essential role in pulmonary surfactant lipid metabolism, lamellar body biogenesis, surfactant protein-B processing, and lung development late in gestation (23). In the current study, ABAC3 expressions were similar in $\mathrm{CDH}$ and non-CDH pups at full term. Controversy persists regarding whether or not humans with $\mathrm{CDH}$ and animal models of $\mathrm{CDH}$ have a surfactant deficiency. Our study revealed that fetal lung maturation was not delayed in $\mathrm{CDH}$ as compared with non-CDH in both humans and the rat nitrofen-induced model of $\mathrm{CDH}$, observations that are consistent with recently reported results $(1,24,25)$.

Our study has limitations. First, the $\mathrm{CDH}$ sample number for the human data was small. To evaluate the efficacy of LBC for predicting lung hypoplasia in $\mathrm{CDH}$, a larger study would be necessary. Next, we must focus on the interpretation of LBC before 35 gestational weeks. LBC was originally a useful predictor for FLM, such that we could not evaluate lung hypoplasia using LBC when the fetal lung was immature. Before 35 gestational weeks, MRI is recommended for predicting lung hypoplasia in $\mathrm{CDH}$. Finally, we dealt with an animal model of lung hypoplasia in $\mathrm{CDH}$ based on the teratogenic effects of nitrofen. A surgical model is potentially suitable for investigating interventional strategies in $\mathrm{CDH}$, and further study would thus be necessary.

In conclusion, $\mathrm{LBC}$ is potentially useful for predicting hypoplastic lung in human $\mathrm{CDH}$ after 35 gestational weeks. LBC has advantages because it can be measured quickly and inexpensively, and it can be objectively determined as an absolute value using a cell counter. This is the first report predicting outcomes of CDH using LBC. Our results also addressed the fact that fetal lung maturation is not delayed in $\mathrm{CDH}$ as compared with controls for human $\mathrm{CDH}$ and for the nitrofen-induced rat $\mathrm{CDH}$ model. Although this study has limitations, we believe that amniotic LBC can serve a new tool for predicting the outcomes and the necessity of neonatal respiratory management of $\mathrm{CDH}$ and would therefore be useful for prenatal counseling.

\section{METHODS}

Case Registration and Amniotic Fluid Collection in Clinical CDH Data were collected from October 2006 to October 2011 at Nagoya University Hospital, Nagoya, Japan. Thirty amniotic fluid samples from $\mathrm{CDH}$ cases were obtained with signed informed consent. All amniotic fluid samples were obtained at cesarean section performed at 27-38 gestational weeks. We collected amniotic fluid by centesis while looking directly at the amniotic bag after incision of the myometrium during cesarean section. Samples were analyzed immediately after arrival at the laboratory without centrifugation, according to a standardized methodology for LBC reported by Neerhof et al. (6). The LBC (per microliter) was determined using a platelet channel on the Sysmex XE-2100 (Sysmex, Kobe, Japan), and the procedure took no more than $30 \mathrm{~min}$. At least $1 \mathrm{ml}$ of amniotic fluid is needed to measure LBC. This study was approved by the ethics committee of Nagoya University Hospital.
Neonatal data such as gestational age at delivery, birth weight, sex, and prognosis including respiratory management (NO and ECMO) were extracted. Fetal growth restriction was defined as birth weight less than the mean - 1.5 SD for gestational age in Japan (26).

Clinical characteristics of the mother such as preeclampsia and diabetes mellitus, both preexisting and gestational (27), were documented for the assessment.

\section{Prognostic Factors in Clinical CDH}

The LHR is the ratio of the right lung area to the head circumference, as described by Metkus et al. (28).

We calculated the percentage of right sided FLV, defined as the ratio of the observed right FLV to the expected right FLV, using MRI (Visart EX 1.5T; Toshiba, Tokyo, Japan), as described by Hayakawa et al. (15). We also evaluated whether the right-sided pulmonary baseline was completely present. Magnetic resonance images with a complete baseline on at least one section were considered to have a complete pulmonary baseline. This finding has been reported to correlate with the severity of $\mathrm{CDH}$ (15). Polyhydramnios was defined as an amniotic fluid index of $>25$ (29).

\section{Nitrofen-Induced Rat CDH Model}

All animal experimental procedures were performed in accordance with the Nagoya University institutional guidelines for animal care, which conform to the National Institutes of Health animal care guidelines. Timed pregnant Sprague-Dawley rats were purchased from Chubu Kagaku Shizai (Nagoya, Japan). Rats were exposed to nitrofen (SigmaAldrich, Tokyo, Japan) on day 9 of pregnancy. Nitrofen $(100 \mathrm{mg})$ was dissolved in $1 \mathrm{ml}$ of olive oil. A rigid metal tube was then inserted into the rat's esophagus to administer the olive oil-nitrofen mixture to the stomach. At E21, we performed cesarean section and collected amniotic fluid and lung tissue from the newborns of both normal rats and those in which $\mathrm{CDH}$ had been induced by nitrofen.

\section{Transmission Electron Microscope}

Materials in fixing solution were centrifuged for $2 \mathrm{~min}$ at $4,000 \mathrm{rpm}$ and then for $4 \mathrm{~min}$ at $6,000 \mathrm{rpm}$. Materials were washed three times in $0.1 \mathrm{~mol} / \mathrm{l}$ phosphate buffer ( $\mathrm{pH} 7.4$ ) for a duration of $10 \mathrm{~min}$ each time and then postfixed with $1 \%$ osmium tetroxide for $1 \mathrm{~h}$. After dehydration with a series of graded ethanol concentrations, ultrathin sections were cut vertically to the epidermal surface on an Ultracut $S$ ultramicrotome (Reichert, Depew, NY). Sections were stained with uranyl acetate and lead citrate and examined with a JEOL-1400EX transmission electron microscope (Jeol, Tokyo, Japan).

\section{Amniotic LBC in the Rat}

We measured amniotic LBC using light microscopy in the rat model, as described by Hunter et al. (21). In summary, a disposable plastic pipette was used to charge amniotic fluid onto a Burker-Turk hemocytometer (ERMA, Tokyo, Japan). The hemocytometer was viewed at $\times 400$, and lamellar bodies were counted for each of the four corners of the BurkerTurk hemocytometer center grid, and the LBC was then divided by 4 .

\section{Western Blotting}

Lung tissues from newborn rats were conserved at $-80^{\circ} \mathrm{C}$ in RNAlater (Qiagen, Tokyo, Japan). Then protein was extracted using protease inhibitor mixture tablets (Roche, Tokyo, Japan). The following antibody dilutions were used for western blot analysis: anti-ABCA3 (antimouse, incubated at 1:1,000 dilution; Covance, Tokyo, Japan), antimouse immunoglobulin G secondary antibodies (Cell Signaling Technology, Tokyo, Japan), and anti- $\beta$-actin (antimouse, incubated at 1:5,000 dilution; Abcam, Cambridge, UK). ABCA3 signals were normalized to $\beta$-actin signals for quantification.

\section{Statistical Analysis}

Statistical analysis of the data was performed with SPSS for Windows (V.19.0; SPSS, Chicago, IL) and Excel for Windows 2010 (Microsoft, Redmond, WA). Differences between the means were assessed using the Mann-Whitney-Wilcoxon test for unpaired samples. Wherever appropriate, Student's $t$-test was also used. A $P$ value $<0.05$ was considered significant. 


\section{ACKNOWLEDGMENT}

The authors thank Yoshikazu Fujita for his valuable technical support.

STATEMENT OF FINANCIAL SUPPORT

No financial assistance was received in support of this study.

\section{REFERENCES}

1. Boucherat O, Benachi A, Chailley-Heu B, et al. Surfactant maturation is not delayed in human fetuses with diaphragmatic hernia. PLoS Med 2007;4:e237.

2. Dubin SB. Characterization of amniotic fluid lamellar bodies by resistivepulse counting: relationship to measures of fetal lung maturity. Clin Chem 1989;35:612-6

3. Lewis PS, Lauria MR, Dzieczkowski J, Utter GO, Dombrowski MP. Amniotic fluid lamellar body count: cost-effective screening for fetal lung maturity. Obstet Gynecol 1999;93:387-91.

4. Grenache DG, Gronowski AM. Fetal lung maturity. Clin Biochem 2006;39:1-10.

5. Piazze JJ, Maranghi L, Cerekja A, et al. Amniotic fluid lamellar body counts for the determination of fetal lung maturity: an update. J Perinat Med 2005;33:156-60.

6. Neerhof MG, Haney EI, Silver RK, Ashwood ER, Lee IS, Piazze JJ. Lamellar body counts compared with traditional phospholipid analysis as an assay for evaluating fetal lung maturity. Obstet Gynecol 2001;97:305-9.

7. Wijnberger LD, Huisjes AJ, Voorbij HA, Franx A, Bruinse HW, Mol BW. The accuracy of lamellar body count and lecithin/sphingomyelin ratio in the prediction of neonatal respiratory distress syndrome: a meta-analysis. BJOG 2001;108:583-8.

8. Weaver TE, Na CL, Stahlman M. Biogenesis of lamellar bodies, lysosomerelated organelles involved in storage and secretion of pulmonary surfactant. Semin Cell Dev Biol 2002;13:263-70.

9. Baath ME, Jesudason EC, Losty PD. How useful is the lung-to-head ratio in predicting outcome in the fetus with congenital diaphragmatic hernia? A systematic review and meta-analysis. Ultrasound Obstet Gynecol 2007;30:897-906.

10. Heling KS, Wauer RR, Hammer H, Bollmann R, Chaoui R. Reliability of the lung-to-head ratio in predicting outcome and neonatal ventilation parameters in fetuses with congenital diaphragmatic hernia. Ultrasound Obstet Gynecol 2005;25:112-8.

11. Arkovitz MS, Russo M, Devine P, Budhorick N, Stolar CJ. Fetal lung-head ratio is not related to outcome for antenatal diagnosed congenital diaphragmatic hernia. J Pediatr Surg 2007;42:107-10; discussion 110-1.

12. Mahieu-Caputo D, Sonigo P, Dommergues M, et al. Fetal lung volume measurement by magnetic resonance imaging in congenital diaphragmatic hernia. BJOG 2001;108:863-8.

13. Gorincour G, Bouvenot J, Mourot MG, et al.; Groupe Radiopédiatrique de Recherche en Imagerie Foetale (GRRIF). Prenatal prognosis of congenital diaphragmatic hernia using magnetic resonance imaging measurement of fetal lung volume. Ultrasound Obstet Gynecol 2005;26:738-44.

14. Walsh DS, Hubbard AM, Olutoye OO, et al. Assessment of fetal lung volumes and liver herniation with magnetic resonance imaging in congenital diaphragmatic hernia. Am J Obstet Gynecol 2000;183:1067-9.

15. Hayakawa M, Seo T, Itakua A, et al. The MRI findings of the right-sided fetal lung can be used to predict postnatal mortality and the requirement for extracorporeal membrane oxygenation in isolated left-sided congenital diaphragmatic hernia. Pediatr Res 2007;62:93-7.

16. Clugston RD, Greer JJ. Diaphragm development and congenital diaphragmatic hernia. Semin Pediatr Surg 2007;16:94-100.

17. Allan DW, Greer JJ. Pathogenesis of nitrofen-induced congenital diaphragmatic hernia in fetal rats. J Appl Physiol 1997;83:338-47.

18. Cilley RE, Zgleszewski SE, Krummel TM, Chinoy MR. Nitrofen dosedependent gestational day-specific murine lung hypoplasia and left-sided diaphragmatic hernia. Am J Physiol 1997;272(2 Pt 1):L362-71.

19. Tenbrinck R, Tibboel D, Gaillard JL, et al. Experimentally induced congenital diaphragmatic hernia in rats. J Pediatr Surg 1990;25:426-9.

20. Sluiter W, Bos AP, Silveri F, et al. Nitrofen-induced diaphragmatic hernias in rats: pulmonary antioxidant enzyme activities. Pediatr Res 1992;32:3948.

21. Hunter LA, McKenna DS, Baptista MA. Comparison of lamellar body counts using light microscopy with standard coulter counter techniques to assess fetal lung maturity. Gynecol Obstet Invest 2006;61:29-33.

22. Ban N, Matsumura Y, Sakai H, et al. ABCA3 as a lipid transporter in pulmonary surfactant biogenesis. J Biol Chem 2007;282:9628-34.

23. Cheong $\mathrm{N}$, Zhang $\mathrm{H}$, Madesh $\mathrm{M}$, et al. ABCA3 is critical for lamellar body biogenesis in vivo. J Biol Chem 2007;282:23811-7.

24. Sullivan KM, Hawgood S, Flake AW, Harrison MR, Adzick NS. Amniotic fluid phospholipid analysis in the fetus with congenital diaphragmatic hernia. J Pediatr Surg 1994;29:1020-3; discussion 1023-4.

25. van Loenhout RB, Tseu I, Fox EK, et al. The pulmonary mesenchymal tissue layer is defective in an in vitro recombinant model of nitrofen-induced lung hypoplasia. Am J Pathol 2012;180:48-60.

26. Yoshida S, Unno N, Kagawa H, Shinozuka N, Kozuma S, Taketani Y. Prenatal detection of a high-risk group for intrauterine growth restriction based on sonographic fetal biometry. Int J Gynaecol Obstet 2000;68:225-32.

27. Sugaya A, Sugiyama T, Nagata M, Toyoda N. Comparison of the validity of the criteria for gestational diabetes mellitus by WHO and by the Japan Society of Obstetrics and Gynecology by the outcomes of pregnancy. Diabetes Res Clin Pract 2000;50:57-63.

28. Metkus AP, Filly RA, Stringer MD, Harrison MR, Adzick NS. Sonographic predictors of survival in fetal diaphragmatic hernia. J Pediatr Surg 1996;31:148-51; discussion 151-2.

29. Moore TR, Cayle JE. The amniotic fluid index in normal human pregnancy. Am J Obstet Gynecol 1990;162:1168-73. 